\title{
Variabilidade e Repetição Operantes Aprendidas após Estímulos Aversivos Incontroláveis ${ }^{1}$
}

\author{
Maria Helena Leite Hunziker ${ }^{2}$ \\ Marcos Takashi Yamada \\ Fernando Nunes Manfré \\ Érika Ferreira de Azevedo \\ Universidade de São Paulo
}

\begin{abstract}
RESUMO - O objetivo desse experimento foi verificar se a experiência prévia com eventos aversivos incontroláveis interfere na aprendizagem da variabilidade ou da repetição operantes. Ratos $(n=45)$ tiveram a reposta de pressão à barra reforçada positivamente em CRF e FR 4, sendo depois divididos em três grupos, expostos a choques elétricos controláveis, incontroláveis ou nenhum choque. Em seguida, receberam nove a 12 sessões de reforçamento positivo para seqüências de quatro respostas de pressão a barra, em uma caixa com duas barras (direita - D e esquerda - E): metade dos sujeitos foi reforçada por variar (VAR) e metade por repetir uma mesma seqüência (REP). Os resultados mostraram que o tratamento prévio com choques não interferiu na aprendizagem dos padrões de variabilidade e de repetição, que foram dependentes apenas da contingência em vigor. Esses dados são contrários ao previsto pela hipótese do desamparo aprendido.
\end{abstract}

Palavras-chave: variabilidade operante; repetição operante; choques incontroláveis; desamparo aprendido.

\section{Operant Variability and Repetition Learned after Uncontrollable Aversive Stimuli}

\begin{abstract}
The aim of the present experiment was to verify whether the previous experience with uncontrollable aversive events interferes in the learning of operant variability and repetition. Rats responses to lever pressure $(n=45)$ were reinforced positively in CRF and FR 4 contingencies and then divided into three groups, exposed to electric shocks that could be either controllable, uncontrollable or none. After that, nine to 12 positive reinforcement sessions were conducted for sequences of four lever pressure responses in a box with two levers (right - D and left - E): half subjects was reinforced for varying (VAR) and half for repeating a same sequence (REP). The results showed that the previous shock treatment did not interfere in the learning of repetition and variability patterns which were only dependent on the contingence in force. These data are in disagreement with the learned helplessness hypothesis prediction.
\end{abstract}

Key words: operant variability; operant repetition; uncontrollable shocks; learned helplessness.

Desde que Darwin desenvolveu a teoria sobre a evolução das espécies, seleção e variação são considerados os mecanismos básicos através dos quais os seres vivos se adaptam ao meio ambiente. No estudo do comportamento, ambos os processos vêm sendo pesquisados, embora com uma grande predileção pelos processos seletivos. Uma evidência disso são os trabalhos de Skinner que destacam a seleção pelas consequências, nos níveis filogenético, ontogenético e cultural (Skinner, 1966; 1981).

Embora o efeito primordial do reforço seja a seleção da classe de respostas que o antecedeu, produzindo a repetição da mesma, sabe-se que padrões variáveis de

1 Esse trabalho foi realizado com o apoio do CNPq (processo no.550987/2002-9) e da FAPESP (processo no. 03/02122-4). Parte desse trabalho foi apresentada na XXXIV Reunião Anual de Psicologia, da Sociedade Brasileira de Psicologia, em Ribeirão Preto, 2004. Os autores agradecem a colaboração de Cristiano Valério dos Santos ao longo de todo o experimento

2 Endereço: Av. Prof. Mello Moraes, 1721, Cidade Universitária, Butantã, São Paulo, SP, Brasil 05508-900. E-mail: hunziker@usp.br respostas também podem ser aprendidos em função de contingências de reforçamento (Neuringer, 2002; 2004). Esse controle operante da variabilidade foi inicialmente demonstrado por Page e Neuringer (1985), que estudaram a sequiência de oito respostas dadas por pombos em dois discos (esquerdo - E e direito - D), sendo uma sequiência reforçada apenas quando fosse diferente das $n$ seqüências anteriores. Para controlar os efeitos da intermitência do reforçamento, esse estudo também utilizou uma contingência de acoplamento: nessa fase, a distribuição dos reforços era a mesma recebida durante o reforçamento da variabilidade, porém contingente apenas à emissão da sequiência, independente da sua variação. Os resultados mostraram que a variabilidade ocorreu de forma acentuada quando foi exigida para liberação do reforço, mas caiu em freqüência na fase de acoplamento, demonstrando o seu controle pelas conseqüências.

A variabilidade comportamental geralmente é considerada sobre diferenças de um comportamento em relação a um outro, chamado de referente (Hunziker \& Moreno, 2000). Ela se relaciona inversamente à previsibilidade do 
comportamento, podendo ser mensurada em graus dentro de um contínuo onde, em um dos extremos tem-se total imprevisibilidade (grau máximo de variação), e no outro a total previsibilidade da ocorrência do comportamento (repetição sistemática do mesmo). Diversos experimentos vêm explorando esse contínuo variabilidade/repetição, demonstrando que ambos os padrões podem ser operantes, ou seja, dependentes do reforçamento contingente a eles (Barba \& Hunziker, 2002; Hunziker, Saldana \& Neuringer, 1996; Machado, 1989; 1993; Neuringer, 1991; 1992; 1993; Stokes, 1995).

Além das contingências em vigor, tem sido demonstrado que a aprendizagem da variação/repetição pode também sofrer interferência da história do sujeito, como, por exemplo, a ordem de apresentação das contingências de acoplamento ou de reforçamento da variabilidade (Hunziker, Caramori, Silva \& Barba, 1998). Contudo, pouco se sabe sobre a interferência de histórias com contingências aversivas sobre essas aprendizagens.

Um dos delineamentos experimentais que tem se mostrado bastante rico na investigação da interferência da história com eventos aversivos sobre novas aprendizagens é o que abrange o efeito chamado de "desamparo aprendido" (learned helplessness). Em estudos-protótipo sobre o desamparo, animais são expostos a duas fases experimentais. Na primeira, os sujeitos são manipulados em trios, sendo dois deles expostos simultaneamente a choques elétricos enquanto o terceiro não é submetido a esses estímulos. Um dos sujeitos pode reduzir a duração dos choques emitindo uma resposta de fuga previamente definida (choques controláveis), enquanto o outro recebe choques similares, porém sem ter a possibilidade de alterar a sua duração (choques incontroláveis). Na segunda fase, todos os sujeitos são submetidos a uma contingência de fuga. Os resultados mostram que apenas os sujeitos previamente expostos aos choques incontroláveis apresentam dificuldade em aprender a resposta de fuga, enquanto os demais aprendem essa resposta rapidamente (Peterson, Maier \& Seligman, 1993; Seligman \& Maier, 1967).

Considerando-se: 1) que a história de exposição a eventos aversivos incontroláveis (não contingentes) geralmente dificulta novas aprendizagens operantes, e 2) que a aprendizagem da variabilidade e da repetição pode ser operante, cabe a pergunta: essa aprendizagem pode ser dificultada pela experiência prévia com estímulos incontroláveis? O presente experimento foi realizado para responder essa pergunta, ou seja, verificar se o mesmo tratamento com choques elétricos incontroláveis, que vêm produzindo sistematicamente o desamparo aprendido em testes de fuga (Hunziker, 2003), pode interferir na aprendizagem da variação e da repetição de seqüências de respostas reforçadas positivamente.

\section{Método}

\section{Sujeitos}

Foram utilizados 48 ratos ingênuos, provenientes do Instituto Butantã, machos, albinos, Wistar, com aproximadamente 90 dias de idade no início do experimento. Os sujeitos foram mantidos em gaiolas individuais, com alimento (ração seca balanceada) e água constantemente disponíveis por um mínimo de uma semana após a sua instalação no biotério do Departamento de Psicologia Experimental da USP. Após esse período de adaptação ao biotério, os animais entraram em regime de privação de água por sete dias antes de iniciar o experimento: o acesso diário à água foi restrito a $10 \mathrm{~min}$ nos primeiros quatro dias, e $5 \mathrm{~min}$ nos dias seguintes. Durante todo o experimento, os animais receberam $3 \mathrm{~min}$ de acesso à água após a sessão. Os animais foram pesados diariamente, para controle da sua saúde, considerando-se requisito para mantê-los no experimento que durante a fase de privação de água não ficassem abaixo de $85 \%$ do seu peso ad lib.

\section{Equipamento}

As sessões que utilizaram choques elétricos foram conduzidas em duas caixas medindo $21,5 \times 21,5 \times 21,0 \mathrm{~cm}$, com a parede frontal de acrílico e as demais de alumínio. O piso da caixa era composto por peças cilíndricas de latão de $0,3 \mathrm{~mm}$ de diâmetro, distando $1,3 \mathrm{~cm}$ entre si. Esse piso estava conectado a um gerador de choques elétricos de corrente alternada com alternador de polaridades (shock scrambler), modelo LVE-133-33, que, quando acionado, produzia a sua eletrificação. No centro da parede lateral direita, a $6 \mathrm{~cm}$ acima do piso, estava localizado um orifício de $3 \mathrm{~cm}$ de diâmetro, que se conectava a uma cuba de alumínio, presa pelo lado externo da caixa, com $3,0 \mathrm{~cm}$ de profundidade. Na parte interna dessa cuba, a $1,5 \mathrm{~cm}$ da borda, havia um sensor elétrico acionado por um feixe de luz (célula foto-elétrica). A interrupção do feixe de luz sobre a célula era registrada como uma resposta. Geralmente isso se dava pela introdução do focinho do animal nesse orifício (focinhador), caracterizando essa resposta como de focinhar.

As sessões com reforçamento positivo foram conduzidas em 12 caixas iguais, cada uma medindo 7,5 X 22,5 $\mathrm{X} 28,0 \mathrm{~cm}$ (comprimento, largura e altura), contendo paredes frontal, traseira e teto em acrílico transparente, sendo as paredes laterais em alumínio. $\mathrm{O}$ piso era composto por barras de metal cilíndricas, de $0,3 \mathrm{~cm}$ de diâmetro, distando $1,3 \mathrm{~cm}$ entre si. Na parede direita essas caixas dispunham de duas barras retangulares de alumínio, medindo 5,0 X 2,0cm (comprimento e largura), ficando a 7,0 cm acima do piso, equiidistantes das laterais, afastadas $9,0 \mathrm{~cm}$ entre si (centro a centro), sendo uma localizada à direita (barra D) e outra à esquerda (barra E). A pressão a qualquer dessas barras, com um mínimo de 45,0gf (grama/força), era registrada como uma resposta de pressão à barra. No nível do piso, centralizado entre as barras, havia um orifício através do qual uma cuba de alumínio podia ser introduzida por acionamento elétrico, disponibilizando ao sujeito $0,05 \mathrm{cc}$ de água. Após 2 s do seu acionamento, a cuba (bebedouro) era rebaixada, ficando fora do alcance do sujeito.

Apenas na fase inicial, foram utilizadas quatro caixas semelhantes às anteriores, com a única diferença que possuíam apenas a barra esquerda. 
Controles e registros foram feitos por computadores PC (386, 486 SX e Pentiun $133 \mathrm{MH}$ ) e softwares desenvolvisdos especialmente para esse experimento em linguagem MEDPC e Delphi.

\section{Procedimento}

\section{Pré-tratamento}

Cada sujeito foi inicialmente submetido ao procedimento de modelagem, por aproximações sucessivas, da resposta de pressão à barra (caixa com uma barra), seguido de 200 reforços liberados em esquema de reforçamento contínuo (CRF). No dia seguinte, o sujeito foi colocado na caixa com duas barras, exigindo-se a emissão de quatro respostas de pressão a qualquer das barras para receber o reforço (FR 4), sendo a sessão encerrada após a liberação de 200 reforços.

\section{Tratamento}

A partir dessa fase, as sessões foram realizas com $24 \mathrm{~h}$ de intervalo, sem interrupção. Os animais foram divididos aleatoriamente em três grupos $(n=16)$ tratados simultaneamente: em cada tríade, dois sujeitos foram colocados na caixa com focinhador, onde receberam 60 choques de $1,0 \mathrm{~mA}$, de $10 \mathrm{~s}$ de duração máxima, liberados a intervalos variáveis de $60 \mathrm{~s}$ (amplitude de variação de 10-110 s); o terceiro sujeito não foi exposto a esses estímulos. Cada choque foi chamado de tentativa, sendo o intervalo entre os choques denominado intervalo entre tentativas (IET). Para um dos grupos, o choque era imediatamente interrompido após a emissão da resposta de focinhar (grupo choque controlável - C), sendo o tempo decorrido entre o início e o término dos choques chamado de latência de fuga. Não havendo a emissão da resposta de fuga, o choque era interrompido automaticamente após $10 \mathrm{~s}$ de seu início, sendo esse tempo registrado como a latência na tentativa. Respostas durante o IET não tinham consequiência programada. Para o segundo grupo, o comportamento do sujeito não produzia mudança na duração dos choques. Esses animais (grupo choque incontrolável - I) compunham pares com os animais $\mathrm{C}$, de forma que, alojados em caixas adjacentes, recebiam ambos os mesmos choques. Portanto, o desligamento dos choques era dependente apenas da resposta de fuga do sujeito do grupo $\mathrm{C}$, sendo independente das respostas do sujeito I, ao qual ele estava acoplado. Os animais do terceiro grupo permaneceram no biotério, sem receber qualquer tratamento (grupo não choque $-\mathrm{N}$ ).

Para analisar a aquisição do comportamento de fuga foram consideradas as latências da resposta de focinhar, agrupadas em blocos de cinco tentativas sucessivas.

\section{Teste}

Nessa fase, a unidade comportamental reforçada foi a seqüência de quatro respostas de pressão à barra, na caixa com duas barras (direta - D e esquerda - E). O reforçamento foi dependente da distribuição das respostas $\mathrm{D}$ ou $\mathrm{E}$ dentro da seqüência, havendo a possibilidade de 16 combinações diferentes (por exemplo, DDDD, EDDE, EEDD, etc). Sequiências reforçadas foram seguidas da apresentação da gota de água e seqüências não reforçadas foram seguidas por um período de 5 s com a luz ambiente apagada (chamado timeout) nas quais nenhum reforço foi apresentado, nem as respostas foram registradas ou consequienciadas.

Cada grupo foi dividido em dois $(n=8)$, sendo metade reforçada por emitir seqüências variáveis (VAR) e metade por emitir seqüências repetidas (REP). Em VAR, a freqüência de emissão de cada seqüência foi considerada proporcionalmente em comparação às demais, sendo condição para reforçamento que ela não ultrapassasse o limiar de 1/16 (ou seja, freqüência relativa de emissão não superior a 0,0625). Além disso, ao ser emitida cada seqüência produzia a multiplicação do fator 0,99 sobre a frequiência das anteriores. Portanto, a cada sequiência emitida, as anteriores tinham reduzido o seu registro de freqüência e aumentada a sua probabilidade de reforçamento. Logo, quanto mais freqüente e recente, menor a probabilidade de reforçamento da sequiência, e vice-versa. Com esse conjunto de exigências, o reforço se tornava mais provável na medida em que todas as 16 sequiências possíveis fossem emitidas, com um mínimo de repetições sucessivas (variabilidade). Cada sessão foi encerrada após a emissão de 200 seqüências ou $45 \mathrm{~min}$, o que ocorresse primeiro. Após a emissão de 150 sequiências, os animais foram submetidos a sete sessões sob a contingência VAR.

Sob a contingência de repetição (REP), o reforço foi liberado apenas após a emissão da sequiência EEEE, sendo 0\% a probabilidade de reforçamento para as demais seqüências. Inicialmente, todas as sequiências EEEE tiveram 100\% de reforçamento. Esse procedimento foi mantido até o sujeito emitir 150 seqüências e atingir $80 \%$ de reforçamento na sessão. No caso de esses critérios não serem atingidos na primeira sessão, o procedimento foi repetido nas sessões seguintes até o sujeito atingir ambos os critérios. A partir daí, a probabilidade de reforçamento para a sequiência EEEE foi reduzida para $50 \%$, permanecendo desta forma por sete sessões consecutivas. Em função desses critérios, os animais tiveram entre 9 a 12 sessões.

A aprendizagem dos padrões de variabilidade ou repetição foi analisada a partir do índice de variabilidade e da taxa de respostas. O índice de variabilidade foi obtido através da medida estatística de distribuição $\mathrm{U}$, extraído da teoria da informação de Attneave (1959). Para tanto, foi utilizada a fórmula $\mathrm{U}=\left(\Sigma\right.$ pi. $\log _{2}$ pi $) / 4$, na qual pi é a probabilidade de emissão de cada seqüência particular, extraída de sua frequiência relativa (ver Barba, 1997). Esse índice pode adquirir valores entre 0,0 e 1,0 indicando, nesses extremos, ausência de variação (repetição) e o máximo de variação possível, respectivamente. Ele foi calculado sobre blocos sucessivos de 200 tentativas: no caso de sujeitos que não atingiram esse número em uma sessão, foram somadas as tentativas de sessões seguintes. Também foram calculadas as taxas de respostas $(\mathrm{R} / \mathrm{min})$ a cada sessão do teste. 
Tabela 1. Composição dos seis grupos experimentais em função dos procedimentos estabelecidos nas fases de tratamento (com estímulo aversivo) e teste (com reforço positivo). Todos os animais passaram previamente por sessão de modelagem de resposta de pressão à barra, reforçamento positivo em CRF e FR 4.

\begin{tabular}{|c|c|c|}
\hline Grupos & $\begin{array}{c}\text { TRATAMENTO } \\
\text { estímulo aversivo } \\
(1 \text { sessão) }\end{array}$ & $\begin{array}{c}\text { TESTE } \\
\text { reforço positivo } \\
\text { (9-12 sessões) }\end{array}$ \\
\hline C-VAR & choque controlável & \multirow{3}{*}{ VARIABILIDADE } \\
\hline I-VAR & choque incontrolável & \\
\hline N-VAR & não choque & \\
\hline C-REP & choque controlável & \multirow{3}{*}{ REPETIÇÃO } \\
\hline I-REP & choque incontrolável & \\
\hline N-REP & não choque & \\
\hline
\end{tabular}

A Tabela 1 resume o procedimento adotado nesse estudo.

Os resultados das fases de tratamento e de teste foram analisados estatisticamente pelos testes ANOVA (simples ou "two-way para medidas repetidas"), teste Tukey para comparação entre duplas e teste $t$ de Student para amostras emparelhadas.

\section{Resultados}

Na fase de tratamento, um dos sujeitos do grupo C não aprendeu a resposta de fuga focinhar. Como era requisito para os sujeitos desse grupo exercer o controle sobre a duração do choque, esse animal foi descartado do experimento juntamente com os seus pares dos grupos I e N. Assim, posteriormente ao tratamento, os grupos REP passaram a contar com $n=7$ na análise dos resultados.

A Figura 1 mostra as latências das respostas de fuga apresentadas pelos animais submetidos aos choques controláveis na fase de tratamento (grupos C), os quais posteriormente foram denominados C-VAR e C-REP: os pontos ligados em linha mostram a latência média do grupo, em blocos de cinco tentativas, enquanto que os pontos soltos indicam as latências individuais. Esses dados mostram que ambos os grupos se comportaram de forma equivalente, com redução das latências ao longo da sessão: na média, as latências finais ficaram em torno de $1 / 4$ do valor das iniciais. Estatisticamente, a ANOVA two way para medidas repetidas confirmou que não houve diferença no padrão apresentado por ambos os grupos, porém foram altamente significantes as diferenças obtidas ao longo dos blocos de tentativas $\left(F_{(11,143)}=47,707, p<0,001\right)$, confirmando a aprendizagem de fuga.

Na fase de teste, a Figura 2 mostra os índices U obtidos pelos sujeitos dos diferentes grupos, sendo que na parte su- perior estão os resultados individuais, ao longo de blocos sucessivos de 200 seqüências, e na parte inferior estão os índices U médios de cada grupo, no primeiro bloco e no total das sessões. A análise dos resultados individuais mostra comportamentos regulares intra-grupo: todos os sujeitos submetidos ao reforçamento da variabilidade (VAR) apresentaram índices $\mathrm{U}$ altos, próximos de 1,0 enquanto que to-

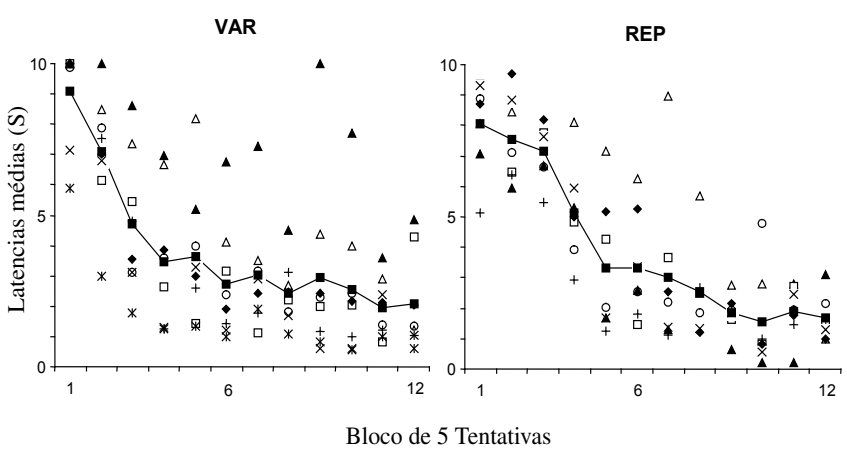

Figura 1. Latências médias (s) da resposta de fuga focinhar, durante a sessão de tratamento, agrupadas em blocos de cinco tentativas. No painel esquerdo estão os resultados dos sujeitos posteriormente expostos à contingência de variabilidade (C-VAR); no painel direito, dados dos sujeitos posteriormente expostos ao reforçamento da repetição (C-REP). Em ambos os painéis, os pontos isolados indicam os resultados individuais e os pontos ligados em linha indicam os dados médios do grupo $(n=8)$.

dos os sujeitos submetidos ao reforçamento diferencial da repetição apresentaram índices $U$ baixos, próximos de 0,0 . Destaque-se que esses padrões opostos foram apresentados desde o primeiro bloco, embora com pequenas diferenças intra-grupo, as quais praticamente desapareceram nos blo$\cos$ finais. Essa regularidade final é especialmente marcante entre os animais submetidos ao reforçamento da repetição, que tiveram todos os índices $\mathrm{U}$ abaixo de 0,1 . Quanto ao tratamento de choques, ele não produziu alteração na aprendizagem da variação ou da repetição: apenas no primeiro 
bloco, os animais expostos aos choques incontroláveis tiveram índice de variabilidade um pouco maior que os demais, tanto quando reforçados a variar como a repetir. Portanto, pode-se dizer que, no geral, esses resultados indicam que os animais apresentaram graus opostos de variabilidade dependendo apenas da contingência de reforçamento em vigor, praticamente sem interferência do tratamento prévio com choques.

Os resultados médios, no primeiro bloco e em todas as sessões, apresentados na parte inferior da Figura 2, refletem esses dados individuais. No total das sessões, apenas a contingência em vigor produziu diferenças marcantes no índice U, com alta variabilidade nos grupos VAR e quase ausência de variabilidade nos grupos REP, independentemente do tratamento prévio com choques. No início da exposição às contingências de reforçamento, o tratamento com choques produziu efeito sutil e transitório: os índices U médios, obtidos no primeiro bloco, mostraram que, independentemente da contingência em vigor, os animais submetidos aos choques incontroláveis apresentaram maior variação que os seus pares expostos aos choques controláveis. Contudo, essas diferenças não foram estatisticamente significantes. A ANOVA indicou que foram significantes apenas as diferenças obtidas no primeiro bloco entre os grupos REP $\left(F_{(2,18)}=3,835, p<0,05\right)$. A comparação entre os pares indicou que essa diferença se deu entre os grupos I e $\mathrm{N}(p<0,05)$. O teste $t$ indicou que houve diferença na comparação entre a primeira sessão e o total das sessões em cada grupo de tratamento $(p<0,01)$.
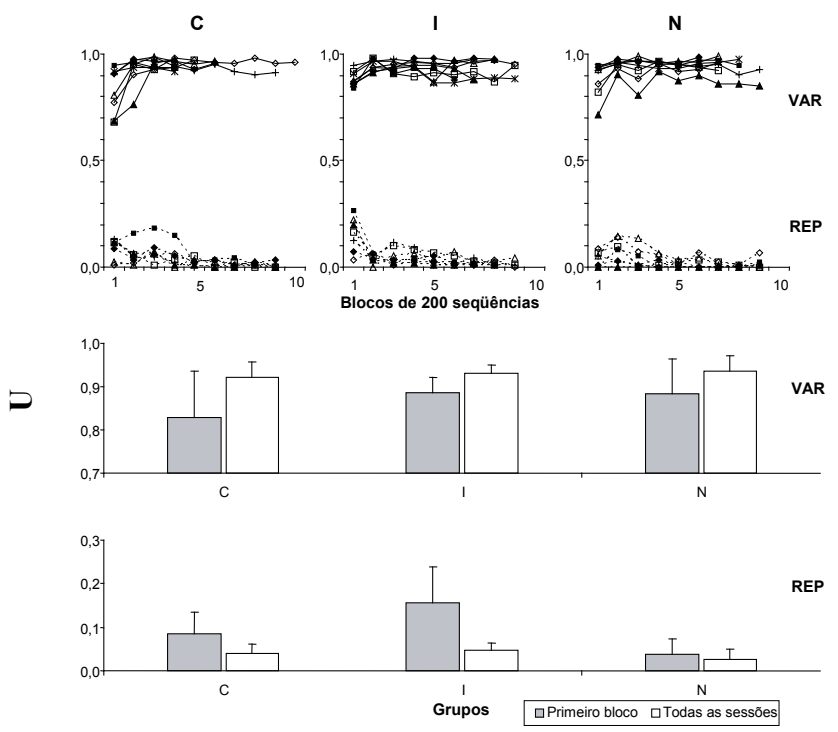

Figura 2. Índices $U$, indicadores do grau de variabilidade das sequiências emitidas pelos sujeitos dos diferentes grupos $(n=8)$. Na parte superior são apresentados dados individuais, agrupados em blocos de 200 sequiências sucessivas. Na parte inferior são apresentados dados médios dos diferentes grupos, relativos a todo o experimento ou apenas ao primeiro bloco de 200 seqüências (notar a diferença na escala das figuras de VAR e REP). As siglas VAR e REP significam que o teste foi realizado sob reforçamento positivo de variabilidade ou repetição, respectivamente; $\mathrm{C}$, I e $\mathrm{N}$ indicam o tratamento prévio com choques controláveis, incontroláveis ou nenhum choque, respectivamente. As linhas verticais no histograma indicam o desvio padrão.
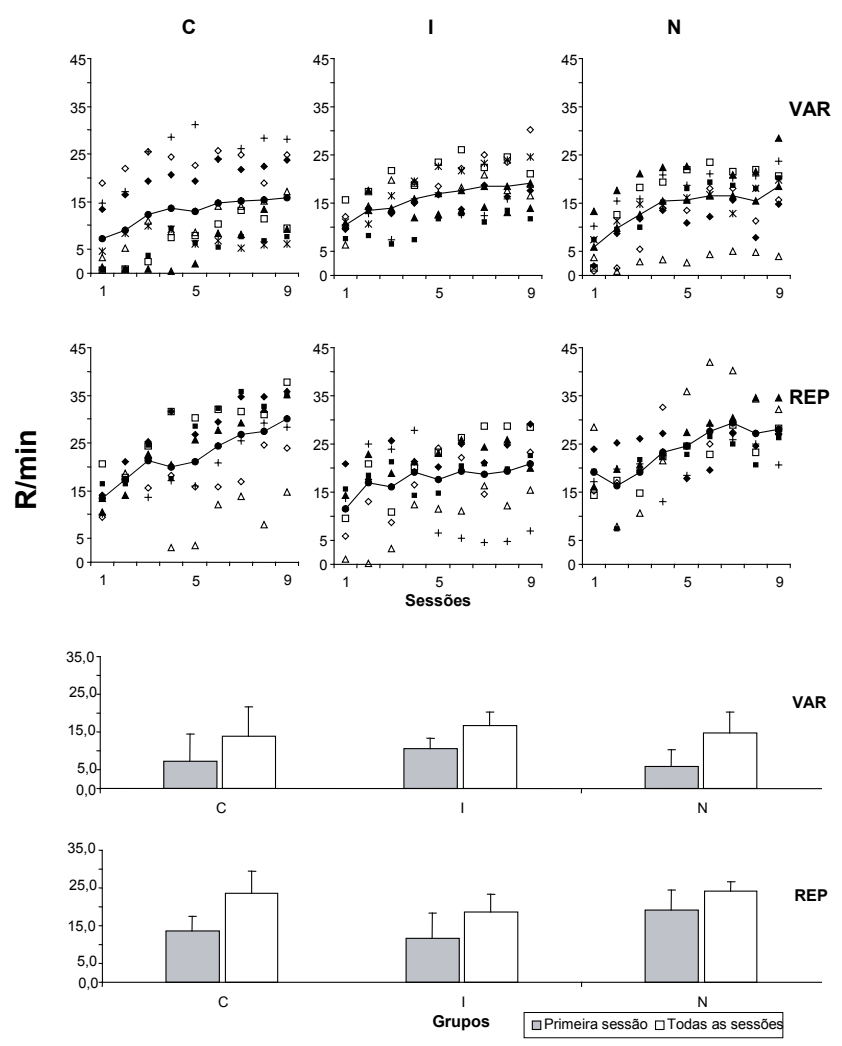

Figura 3. Taxa média de respostas $(\mathrm{R} / \mathrm{min})$ apresentada pelos sujeitos dos diferentes grupos $(n=8)$ nas primeiras nove sessões de teste. Na parte superior, o gráfico de linha indica a média do grupo e os pontos soltos mostram os dados individuais, agrupados por sessão. Na parte inferior são apresentados dados médios dos diferentes grupos, relativos a todo o experimento ou apenas à primeira sessão. As siglas VAR e REP significam, respectivamente, que o teste foi realizado sob reforçamento positivo de variabilidade ou repetição; as letras $\mathrm{C}, \mathrm{I}$ e N indicam o tratamento prévio com choques controláveis, incontroláveis ou nenhum choque, respectivamente. As linhas verticais no histograma indicam o desvio padrão.

A taxa de respostas não sofreu efeito da contingência em vigor nem do tratamento prévio com choques, sendo aparentemente afetada apenas pela quantidade de exposição ao reforçamento. Na parte superior da Figura 3, os dados individuais mostram que todos os sujeitos aumentaram suas taxas na medida em que foram expostos às sessões sucessivas, independentemente da contingência em vigor ou do tratamento prévio com choques. Na parte inferior dessa figura, as médias dos grupos mostraram que não houve diferença sistemática entre os diferentes grupos, porém houve aumento da taxa no total das sessões, comparativamente à primeira. Estatisticamente, a ANOVA indicou que foram significantes apenas as diferenças entre os grupos REP no primeiro bloco $\left(F_{(2,18)}=3,835, p<0,05\right)$, não sendo significantes as diferenças obtidas ao longo de todas as sessões. A comparação entre os pares indicou que essa diferença se deu entre os grupos I e $\mathrm{N}(p<0,05)$. O teste $t$ indicou que houve diferença na comparação entre a primeira sessão e o total das sessões em cada grupo de tratamento $(p<0,01)$.

Mesmo com os valores de $\mathrm{U}$ altos, os animais VAR emitiram seqüências que não atenderam as exigências da contingência, de forma que, ao longo de todo o experimento, cerca de metade das seqüências emitidas foram reforçadas, 
equiparando-se aos sujeitos REP, que receberam 50\% de reforçamento.

\section{Discussão}

Os resultados da sessão de tratamento mostraram que o comportamento de focinhar foi modificado pelas suas consequiências: essa resposta se tornou mais freqüente e mais rápida na medida em que o sujeito foi exposto à remoção do choque contingente a ela. Se por um lado esse padrão de emissão de respostas caracteriza o controle operante estabelecido pelo reforçamento negativo (Catania, 1998), por outro ele também demonstra o controle que os sujeitos dos grupos $\mathrm{C}$ exerceram sobre a duração dos choques. Portanto, essa fase estabeleceu a condição necessária ao experimento que era expor os sujeitos dos grupos $\mathrm{C}$ e I a choques equivalentes, porém possibilitando o controle sobre a sua duração apenas aos sujeitos $\mathrm{C}$.

$\mathrm{Na}$ fase de teste, os níveis de variação das seqüências foram dependentes apenas das contingências de reforçamento: os níveis de variabilidade foram altos quando o reforço seguiu seqüências que diferiam das anteriores (grupos VAR), porém foram baixos quando se reforçou a repetição de uma única sequiência (grupos REP). Essa alta replicabilidade intra-grupo, obtida desde os primeiros blocos de tentativas com todos os sujeitos, fortalece a demonstração experimental sobre a função operante da variação e da repetição aqui registradas, confirmando o que vem sendo descrito na literatura (Page \& Neuringer, 1985; Neuringer, 2002; 2004).

Como dado original desse experimento, tivemos que o controle operante dos diferentes níveis de variação não foi afetado pela história prévia com estímulos aversivos incontroláveis (conforme visto na Figura 2). Tais resultados contrariam o que vem sendo amplamente descrito nos estudos sobre desamparo aprendido, ou seja, dificuldade de aprendizagem operante após a exposição a eventos aversivos incontroláveis (Maier \& Seligman, 1976; Peterson \& cols., 1993). Por que a aprendizagem operante da variação e da repetição não sofreu a interferência dos choques incontroláveis apresentados previamente?

Conforme analisado por Capelari e Hunziker (2005), o uso do reforço positivo no teste é uma variável que pode ser crítica, uma vez que a maioria das pesquisas sobre o desamparo utiliza apenas estímulos aversivos nas diferentes fases experimentais (Maier \& Seligman, 1976; Peterson \& cols., 1993). O uso de choques elétricos no tratamento e água no teste, conforme feito no presente experimento, acarreta, ao menos, duas diferenças básicas em relação à maioria dos estudos sobre desamparo: a natureza aversiva/apetiviva dos estímulos e a não similaridade física entre eles. Embora alguns autores tenham descrito desamparo em contextos envolvendo estímulos não aversivos (por exemplo, Enberg, Welker, Hansen \& Thomas, 1972; Rosellini, 1978), outros estudos descreveram ausência do desamparo quando manipularam arranjos combinando estímulos aversivos/apetitivos no tratamento e teste (Capelari \& Hunziker, 2005;
Mauk \& Pavur, 1979; Rapaport \& Maier, 1978). Uma possível explicação, proposta por Capelari e Hunziker (2005) para a não ocorrência do desamparo nesse tipo de arranjo experimental, é que a aprendizagem que se estabelece na fase de incontrolabilidade só se generaliza para o teste se ambas as fases envolverem estímulos semelhantes entre si. Essa interpretação leva em conta o princípio de generalização de estímulos, segundo o qual quanto mais semelhantes forem os estímulos em diferentes condições de aprendizagem mais provável é que a aprendizagem estabelecida em uma condição se generalize para a outra (Catania, 1998). Dessa perspectiva, a aprendizagem de impossibilidade de controle, estabelecida durante os choques incontroláveis, tem baixa probabilidade de se generalizar para contingências que envolvem reforçamento positivo.

Poucos experimentos investigaram o desamparo com diferentes estímulos aversivos, no tratamento e teste. Uma das raras exceções é o trabalho de Lee e Maier (1988) que analisaram os efeitos de choques incontroláveis sobre a posterior aprendizagem de fuga em uma caixa com água (shuttlebox aquática). As revisões de área mostram que a imensa maioria dos trabalhos sobre desamparo aprendido, realizados com ratos como sujeitos, utiliza como estímulo aversivo apenas o choque elétrico (Peterson \& cols., 1993; Hunziker, 2005). Possivelmente, a dificuldade técnica de se utilizarem outros estímulos aversivos é que tem mantido esse arranjo predominante. Porém, dada a questão teórica envolvida - sobre a generalização, para o teste, da aprendizagem estabelecida no tratamento - é especialmente importante que alguns pesquisadores estejam buscando soluções técnicas para o estudo do controle aversivo com outros estímulos que não o choque elétrico, tais como o jato de ar quente (Carvalho Neto \& cols., 2005). Na medida em que novos estímulos aversivos sejam facilmente manipuláveis no laboratório com animais, será possível testar essa dissociação entre a similaridade física e a funcional dos estímulos, identificando a sua relevância na determinação do desamparo. A priori, não se pode dizer que essas duas interpretações sejam excludentes, podendo haver uma somatória dos efeitos da semelhança dos estímulos com a sua função aversiva ou apetitiva. Contudo, apenas novos experimentos, delineados especialmente para esse fim, permitirão o avanço dessa análise. Mesmo sem poder separar essas duas variáveis, contudo, pode-se afirmar que os resultados aqui obtidos mostraram que a aprendizagem da variabilidade e da repetição, com reforço positivo, não foi afetada pela experiência prévia com choques elétricos incontroláveis.

Não se pode deixar de analisar o fato de que o teste utilizado no presente estudo difere da maioria dos trabalhos sobre desamparo quanto à complexidade da contingência de reforçamento e ao número de sessões. Geralmente, a aprendizagem no teste é avaliada em uma única sessão de fuga com contingência simples, na qual a emissão de apenas uma resposta interrompe o choque. Aqui, a aprendizagem no teste foi avaliada ao longo de nove a 12 sessões de reforçamento positivo, sendo a unidade comportamental uma seqüência de quatro respostas, com reforçamento contingente à configuração da sequiência. Contudo, se a maior complexidade da con- 
tingência no teste fosse uma variável crítica para a ocorrência do desamparo, os resultados aqui obtidos já deveriam ter sido diferentes entre os grupos REP e VAR: em REP a sequiência exigida para reforçamento era simples (quatro respostas na barra esquerda), enquanto em VAR a exigência era diferente a cada nova seqüência emitida, tendo-se como referente de comparação as sequiências emitidas anteriormente. Contudo, apesar dessa diferença em termos da complexidade da contingência, os resultados entre VAR e REP foram iguais, ou seja, ausência de desamparo. Nesse sentido, o atual experimento se soma ao de Capelari e Hunziker (2005), mo qual o comportamento de pressão à barra ficou sob controle do reforço positivo, sob um esquema múltiplo-concorrente, independentemente dos sujeitos terem sido previamente expostos a choques controláveis, incontroláveis ou nenhum choque.

Apesar desse resultado aparentemente contrário ao esperado pelos estudos do desamparo aprendido, deve-se considerar o efeito, pequeno e transitório, obtido nos primeiros momentos de teste que, embora não significante estatisticamente, ocorreu tanto no presente estudo como no de Capelari e Hunziker (2005). Será que o desamparo é uma interferência que se dá apenas nos primeiros momentos de uma nova condição de aprendizagem, não perdurando se houver exposição continuada à nova contingência? Se assim for, então o número de sessões no teste pode ser uma variável relevante no estudo do desamparo. Como, no geral, os estudos com desamparo testam seus sujeitos em apenas uma sessão de fuga, essa pergunta também requer mais experimentos para identificar a relevância dessa variável. Por exemplo, nos estudos que utilizam apenas choques elétricos em ambas as fases, os sujeitos deveriam ser submetidos repetidas vezes ao teste de fuga para identificar se a dificuldade de aprendizagem é mantida ou se ao longo de diversas exposições o comportamento do sujeito passa a ficar sob o controle do reforçamento negativo. Contudo, ao se comparar os estudos com estímulos aversivos com os que utilizam estímulos apetitivos, é necessário considerar que sessões sucessivas de reforçamento positivo não são equivalentes a sessões sucessivas de fuga: em testes com reforçamento positivo, não emitir a resposta tem como conseqüência apenas a não obtenção do reforço; nas sessões de fuga, não emissão da resposta tem como conseqüência o sujeito continuar recebendo o choque, que é desligado automaticamente após seu tempo de duração máxima (ou seja, independentemente da resposta). Portanto, testes repetidos de fuga, com sujeitos que apresentam o desamparo, aumentam a sua exposição a choques incontroláveis. Essa diferença dificulta a comparação direta dos estudos que utilizam testes repetidos de aprendizagem com reforçamento positivo ou negativo. Serão necessários novos estudos, delineados especificamente com o fim de fazer essa comparação, para que se possa identificar a relevância do número de sessões no teste nos estudos sobre o desamparo aprendido.

Por fim, a não ocorrência do desamparo no presente estudo poderia ser atribuída à fase pré-tratamento que permitiu ao animal exercer controle sobre o reforço positivo. Diversos estudos vêm mostrando que uma primeira experiência com estímulos controláveis pode impedir que choques incontro- láveis produzam posteriormente o desamparo, efeito esse denominado "imunização" (Seligman, Rosellini \& Kozak, 1975). Contudo, os estudos que relataram imunização utilizaram estímulos aversivos em todas as fases do experimento (Alloy \& Bersh, 1979; Hannum, Rosellini \& Seligman, 1976; Seligman \& cols, 1975; Yano \& Hunziker, 2000), de forma que o animal aprendia que choques eram controláveis antes de se submeter aos incontroláveis. Os estudos que utilizaram reforço positivo antes dos choques incontroláveis, em esquemas simples e por poucas sessões como o aqui feito, não têm produzido imunização no teste de fuga. Por exemplo, Mestre e Hunziker, (1996) obtiveram desamparo no teste com ratos que haviam sido expostos a uma sessão de reforçamento positivo (CRF) antes dos choques incontroláveis. Em outro estudo, um número maior de sessões (cinco a 12 sessões) com diferentes contingências de reforço positivo (CRF, FR 6 ou múltiplo FR/extinção), antes dos choques incontroláveis, produziu imunização apenas parcial em testes de fuga (Hunziker \& Lima, no prelo). Com base nesses estudos, consideramos que é pouco provável que os reforços positivos, aqui utilizados na fase pré-tratamento, tenham produzido a imunização ao desamparo.

Portanto, o presente experimento indicou que, dentro dos parâmetros aqui utilizados, a experiência prévia com choques incontroláveis não interferiu na aprendizagem da variabilidade/repetição operantes, reforçadas positivamente. Esses resultados confirmam a relevância das contingências operantes no controle desses padrões opostos de variabilidade (Neuringer, 2002; 2004), a despeito das diferentes histórias dos sujeitos envolvendo experiências de controle, ou impossibilidade de controle, sobre estímulos aversivos.

\section{Referências}

Alloy, L. B. \& Bersh, P. J. (1979). Partial control and learned helplessness in rats: Control over shock intensity prevents interference with subsequent escape. Animal Learning and Behavior, 7, 157-164.

Attneave, F. (1959). Applications of information theory to psychology: A summary of basic concepts, methods and results. New York: Holt-Dryden Book.

Barba, L. S. (1997). Variabilidade Comportamental Aprendida. Dissertação de Mestrado, Universidade de São Paulo, São Paulo.

Barba, L. S. \& Hunziker, M. H. L. (2002). Variabilidade comportamental produzida por dois esquemas de reforçamento. Acta Comportamentalia, 10, 5-22.

Capelari, A. \& Hunziker, M.H.L. (2005). Aprendizagem de fuga após estímulos apetitivos incontroláveis. Psicologia: Teoria e Pesquisa, 21, 99-107.

Catania, A. C. (1998). Learning. Englewood Cliffs. New Jersey: Prentice-Hall, Inc.

Carvalho Neto, M. B., Maestri, T. C., Ribeiro; T. C.,Coutinho, E., Miccione, M, Oliveira, R. C. V., Ferreira, F., Faria, D. C. , Moreira, D. E., Tobias, G. K. (2005). O jato de ar quente como estímulo punidor em rattus norvergicus. Psicologia: Teoria e Pesquisa, 21(3), 335-339. 
Enberg, L. A., Welker. R. L., Hansen., G. \& Thomas, D. R. (1972). Acquisition of key pecking via auto shaping as a function to prior experience: "Learned Laziness"? Science, 178, 10021004.

Hannum, R.D., Rosellini, R. \& Seligman, M.E.P. (1976). Learned helplessness in the rat: retention and immunization. Developmental Psychology, 12, 449-454.

Hunziker, M. H. L. (2003). Desamparo aprendido. Tese de LivreDocência, Universidade de São Paulo, São Paulo.

Hunziker, M. H. L. (2005). O desamparo aprendido revisitado: estudos com animais. Psicologia: Teoria e Pesquisa, 21(2), 131-139.

Hunziker, M. H.L., Caramori, F. C., Silva, A. P. \& Barba, L. S. (1998). Efeitos da história de reforçamento na variabilidade comportamental. Psicologia: Teoria e Pesquisa, 14(2), 149-159.

Hunziker, M. H. L. \& Lima, R. S. G. G. (no prelo). Efeito de imunização após reforçamento positivo em ratos. Interação em Psicologia.

Hunziker, M. H. L. \& Moreno, R. (2000). Análise da noção de variabilidade comportamental. Psicologia: Teoria e Pesquisa, 16(2), 135-143.

Hunziker, M. H. L., Saldana, L. \& Neuringer, A. (1996). Behavioral variability in SHR and WKY rats as a function of rearing environment and reinforcement Contingency. Journal of Experimental Analysis of Behavior, 65, 129-144.

Lee, R. K. K. \& Maier, S. F. (1988). Inescapable shock and attention to internal versus external cues in a water escape discrimination task. Journal of Experimental Psychology: Animal Behavior Processes, 14, 302-311.

Machado, A. (1989). Operant conditioning of behavior variability using a percentile reinforcement schedule. Journal of the Experimental Analysis of Behavior, 52, 155-166.

Machado, A. (1993). Learning variable and stereotypical sequences of responses: some data and new model. Behavioral Processes, 30, 103-130.

Maier, S. F. \& Seligman, M. E. P. (1976). Learned helplessness: Theory and evidence. Journal of Experimental Psychology: General, 105, 3-46.

Mauk, M. D. \& Pavur, E. J. (1979). Interconsequence generality of learned helplessness. Bulletin of the Psychonomic Society, 14, 421-423.

Mestre, M. B. A. \& Hunziker, M. H. L. (1996). O desamparo aprendido, em ratos adultos, como função de experiências aversivas incontroláveis na infância. Tuiuti: Ciência e Cultura, 6(2), 25-47.
Neuringer, A. (1991). Operant variability and repetition as functions of interesponse time. Journal of Experimental Psychology: Animal Behavior Processes, 17, 3-12.

Neuringer, A. (1992). Choosing to vary and repeat. Psychological Science, 3, 246-250.

Neuringer, A. (1993). Reinforced variation and selection. Animal Learning and Behavior, 21(2), 83-91.

Neuringer, A. (2002). Operant variability: Evidence, function, and theory. Psychonomic Bulletin \& Review, 9, 672-705.

Neuringer, A. (2004). Reinforced variability in animals and people: Implications for adaptative action. American Psychologist, 59, 891-906.

Page, S. \& Neuringer, A. (1985). Variability is an operant. Journal of Experimental Psychology: Animal Behavior Processes, 11, 429-452.

Peterson, C., Maier, S. F. \& Seligman, M. E. P. (1993). Learned helplessness: a theory for the age of personal control. New York: Oxford University Press.

Rapaport, P. M. \& Maier, S. F. (1978). Inescapable shock and food-competititon dominance in rats. Animal Learning and Behavior, 6, 160-165.

Rosellini, R. A. (1978). Inescapable shock interferes with the acquisition of an appetitive operant. Animal Learning and Behavior, 7, 327-339..

Seligman, M. E. P. \& Maier, S. F. (1967). Failure to escape traumatic shock. Journal of Experimental Psychology, 74, 1-9.

Seligman, M. E. P., Rosellini, R. A. \& Kozak, M.J. (1975). Learned helplessness in the rat: time course, immunization, and reversibility. Journal of Comparative and Physiological Psychology, $88,542-547$.

Skinner, B. F. (1966). The phylogeny and ontogeny of behavior. Science, 153, 1204-1213.

Skinner, B. F. (1981). Selection by consequences. Science, 213, 501-504.

Stokes, P. (1995). Learned variability. Animal Learning and Behavior, 23, 164-176.

Yano, Y. \& Hunziker, M. H. L. (2000). Desamparo aprendido e imunização com diferentes respostas de fuga. Acta Comportamentalia, 8, 143-166.

Recebido em 23.02.2006

Primeira decisão editorial em 09.03.2006

Versão final em 05.06.2006

Aceito em 14.07.2006 